\title{
BMJ Open Self-reported sleep in late pregnancy in relation to birth size and fetal distress: the E Moe, Māmā prospective cohort study
}

\author{
Laura D Howe, ${ }^{1,2}$ T Leigh Signal, ${ }^{2}$ Sarah-Jane Paine, ${ }^{2}$ Bronwyn Sweeney, ${ }^{2}$ \\ Monique Priston, ${ }^{2}$ Diane Muller, ${ }^{2}$ Kathy Lee, ${ }^{3}$ Mark Huthwaite, ${ }^{4}$ Philippa Gander ${ }^{2}$
}

To cite: Howe LD, Signal TL, Paine S-J, et al. Self-reported sleep in late pregnancy in relation to birth size and fetal distress: the E Moe, Māmā prospective cohort study. BMJ Open 2015;5:e008910. doi:10.1136/bmjopen-2015008910

- Prepublication history and additional material is available. To view please visit the journal (http://dx.doi.org/ 10.1136/bmjopen-2015008910).

Received 27 May 2015 Revised 11 August 2015 Accepted 19 August 2015

CrossMark

\footnotetext{
${ }^{1}$ School of Social and Community Medicine, University of Bristol, Bristol, UK

${ }^{2}$ Sleep-Wake Research Centre, Massey University, Wellington, New Zealand ${ }^{3}$ University of California, San Francisco, California, USA

${ }^{4}$ University of Otago, Wellington, New Zealand
}

Correspondence to Dr Laura D Howe; laura.howe@bristol.ac.uk

\section{ABSTRACT}

Objectives: To explore associations between features of sleep during pregnancy and adverse outcomes for the infant.

Setting: E Moe, Māmā is a cohort study in Aotearoa/ New Zealand that investigates self-reported sleep and maternal health in late pregnancy and the postpartum period.

Participants: Women (N=633; 194 Māori) reported detailed information on their sleep duration, quality, disturbances, disorders (snoring, breathing pauses, twitching legs, restless legs) and daytime sleepiness between 35 and 37 weeks gestation.

Outcome measures: Birthweight and fetal distress during labour were extracted from medical records. Associations between each sleep variable and small or large for gestational age (SGA/LGA) using customised birthweight centile or fetal distress were estimated using multinomial/logistic regression, controlling for potential confounders. Secondary analyses considered differences in associations between Māori and nonMāori women.

Results: There was some indication that breathing pauses (a measure of sleep apnoea) were associated with both SGA (OR 2.8, 95\% Cl 0.9 to 9.0, $\mathrm{p}=0.08$ ) and LGA (OR 2.0, $95 \% \mathrm{Cl} 0.7$ to $5.7, \mathrm{p}=0.20$ ), with the association for LGA being stronger when only pregnancy-onset breathing pauses were considered (OR 3.5, 95\% Cl 1.3 to 9.6, $\mathrm{p}=0.01$ ). There was also some evidence that pregnancy-onset leg twitching $(O R$ $3.3,95 \% \mathrm{Cl} 1.1$ to $10.0, p=0.03$ ) and frequent sleep disturbance due to feeling too hot or too cold (OR 1.7, $95 \% \mathrm{Cl} 0.9$ to $3.6, p=0.13$ ) were associated with higher risk of fetal distress. Other sleep measures, including snoring, were not associated with SGA, LGA or fetal distress. Many of the associations we observed were considerably stronger in Māori compared with non-Māori women.

Conclusions: We did not find evidence of previously reported associations between snoring and SGA. Our findings tentatively suggest that self-reported breathing pauses and leg twitching in late pregnancy are associated with infant outcomes, and highlight ethnic inequalities.

\section{Strengths and limitations}

- This study used a broad range of measures of sleep in pregnancy, and looked at their associations with objective measures of birth weight and fetal distress, obtained from medical records.

- Importantly, we found ethnic differences in the associations, with stronger associations between sleep in pregnancy and fetal outcomes in women identifying as Maori.

- Important limitations of this study include the self-reported nature of sleep during pregnancy, and the lack of preterm births in this cohort (due to recruitment in late pregnancy).

\section{BACKGROUND}

Lower birthweight and other birth complications have considerable short-term and longterm consequences for the infant. ${ }^{1-3}$ While multiple risk factors for lower birthweight and birth complications have been identified, ${ }^{4}$ their predictive power remains modest, ${ }^{5}$ and there is therefore high potential benefit in identifying novel risk factors for birth complications. Despite approximately one-third of a person's life being spent asleep, the overwhelming majority of research into the potential causes of birth complications has focused on non-sleeprelated factors. ${ }^{6}$ Sleep disturbance, changes to sleep duration and reduced sleep quality are common during pregnancy, ${ }^{6-15}$ and given the large body of evidence suggesting links between sleep and other health outcomes, ${ }^{16-25}$ investigating their role in the aetiology of birth complications is important.

There is emerging evidence that sleep changes during pregnancy may be associated with pregnancy and birth outcomes. ${ }^{6} 2627$ Several studies have shown an association between poor sleep quality or short sleep 
duration during pregnancy and preterm birth, ${ }^{12} 142829$ with inflammatory mechanisms proposed as a potential explanation (eg, disturbed sleep leading to disruptions to normal immune processes).$^{13}$ Some, though not all, ${ }^{30}$ studies have also suggested a link between sleep during pregnancy and fetal growth including identifying snoring as a risk factor for small for gestational age (SGA) infants. ${ }^{29} 31$ The majority of the existing studies on this topic, however, have a small sample size, with only two recent studies having larger samples. ${ }^{29}{ }^{31}$ Most, though not all, ${ }^{31}$ studies also concentrate on preterm birth and SGA, and do not examine potential associations between sleep during pregnancy and large for gestational age (LGA) infants or other infant outcomes. Furthermore, most focus only on a small number of facets of sleep, ignoring the complex nature of sleep and the possibility for multiple dimensions to be important determinants of birth complications.

In this paper, we use data from 633 participants of a prospective cohort study of pregnant women in Aotearoa/New Zealand (NZ) to examine the associations of multiple facets of sleep in late pregnancy (sleep duration, sleep quality, sleep disturbances, sleep disorders and daytime sleepiness) with fetal distress and infant birth weight. In NZ, the Māori people are the indigenous population. Stark health inequalities exist in NZ, with Māori people suffering from worse health outcomes than non-Māori people, including higher rates of SGA infants. ${ }^{32}$ Therefore, as a secondary aim, we also examined whether the associations between sleep during pregnancy and infant outcomes differed between the 194 Māori participants and the 439 non-Māori participants.

\section{METHODS}

\section{Participants}

The E Moe, Māmā: Maternal Sleep and Health in Aotearoa/NZ study (N=1186; 423 Māori and 763 non-Māori recruited October 2009-October 2011) surveyed a community sample of women during the third trimester of pregnancy (approximately 35-37 weeks gestation) who were carrying a single fetus. This sample thus excludes the majority of preterm births. Equal numbers of Māori and non-Māori were sought in recruitment. ${ }^{33}$

\section{Measurement of maternal sleep parameters}

Maternal sleep in late pregnancy was assessed by selfcompleted questionnaire. Women were asked to report the average number of hours sleep in $24 \mathrm{~h}$ (including naps) during the past week, and before this pregnancy. This was categorised as short sleep $(\leq 6 \mathrm{~h})$, average sleep $(>6$ to $<9 \mathrm{~h})$ or long sleep $(\geq 9 \mathrm{~h})$. We also defined change in sleep duration from pre-pregnancy to late pregnancy, based on movement between these categories from pre-pregnancy to late pregnancy.

Pre-pregnancy and late pregnancy poor quality sleep was defined as not getting a good night's sleep on three or more nights per week, based on one of the Diagnostic and Statistical Manual of Mental Disorders, Fourth Edition (DSM-IV) criteria for insomnia. Late pregnancy sleep quality was also assessed by the General Sleep Disturbance Scale (GSDS), which was used as a total score, and separate subscale scores for sleep quality and daytime sleepiness. Each of these scores was dichotomised into problematic and non-problematic (mean item scores $\geq 3$ or $<3$, respectively) sleep. ${ }^{34} 35$

Women were asked to report the number of nights in the previous week on which a range of factors, many associated with pregnancy, had disturbed their sleep; for each factor we considered three or more disturbed nights as problematic. ${ }^{36}$

The Epworth Sleepiness Scale (ESS ${ }^{37}$ was used to assess daytime sleepiness, with scores greater than 10 indicating excessive daytime sleepiness.

Three separate symptoms of sleep disorders were investigated: snoring, breathing pauses during sleep and leg twitching/jerking. Each symptom was considered to be present if women reported that it occurred $\geq 3$ times per week during late pregnancy. ${ }^{38}$ For each of these sleep symptoms, we also defined 'pregnancy-onset' sleep problems as being present in women who reported the symptoms on $\geq 3$ nights per week during late pregnancy, but on $<3$ nights per week pre-pregnancy. For snoring, we additionally defined 'chronic snoring' as snoring on $\geq 3$ nights per week in pre-pregnancy as well as late pregnancy.

As a separate sleep disorder, restless legs was defined as a positive response to four questions: ever experiencing an urge to move legs (usually accompanied by unpleasant sensations), and whether this is worse at night, more noticeable when resting, and relieved by movement. ${ }^{39}$

\section{Measurement of infant outcomes}

Infant birth weight and gender were extracted from medical records by the District Health Boards/Ministry of Health through matching on the infant's national health index number and birth date. Customised birthweight centiles were generated using the Grow software for the NZ population (using software developed by the Gestation Network, available from https://www. gestation.net/cc/about.htm. Access date 23 September 2015). ${ }^{40}$ This method develops birthweight centiles accounting for maternal height, weight, ethnicity, parity and the infant's sex and gestation at delivery. This method is intended to separate pathological from constitutionally small babies, and has been shown to result in stronger associations between small size at birth and perinatal mortality compared with uncustomised birthweight centiles. ${ }^{41}{ }^{42}$ We defined SGA as $<10$ th customised centile, appropriate for gestational age (AGA) as 10-90th centile and LGA as $>90$ th centile.

Fetal distress refers to a range of symptoms either during pregnancy or delivery that suggests the fetus may not be well. Fetal distress was defined as one or more of the following the International Classification of Diseases 
(ICD) $10^{43}$ codes being recorded in the obstetric records (ICD 10 codes shown in parentheses): maternal care for signs of hypoxia (O363), labour and delivery complicated by fetal heart rate anomaly $(\mathrm{O} 680)$, fetal heart rate anomaly with meconium in amniotic fluid (O682), biochemical evidence of fetal stress (O683) or other evidence of fetal stress (O688).

\section{Measurement of potential confounders}

Maternal age, parity and self-identified ethnicity were recorded in the self-completed questionnaire in late pregnancy; any woman identifying as Māori as a single response, or one of many choices of ethnicity, was classified as Māori. Everyone else was classified as non-Māori. This approach is the recommended standard for health research in NZ. ${ }^{44}$ Maternal smoking was categorised as 'smokers' (regular or occasional smokers) and 'nonsmokers' (ex-smokers or non-smokers). Height and prepregnancy weight reported in the questionnaire were used to calculate pre-pregnancy body mass index (BMI; $\mathrm{kg} / \mathrm{m}^{2}$ ). Area-level deprivation was measured using the NZ Deprivation Index 2006 (NZDep), ${ }^{45}$ a measure that assigns households to a 10th of deprivation based on residential address. Maternal depressive symptoms were assessed in late pregnancy using the Edinburgh Postnatal Depression Scale (EPDS). ${ }^{46}$ Gestational age at delivery was reported by the mothers in a questionnaire at 12 weeks postpartum.

\section{Statistical analysis}

Analysis was restricted to participants with data on all of the measures of sleep in late pregnancy, customised birthweight centile, fetal distress, infant gender and all a priori determined potential confounders (prepregnancy sleep duration and number of nights good sleep, maternal age, parity, ethnicity, pre-pregnancy BMI, smoking (any vs none), NZ Deprivation Index, gestational age at delivery and maternal EPDS score).

Our primary aim was to determine the association between maternal sleep and infant outcomes in the whole population (Māori and non-Māori combined). Associations between each sleep measure and categories of customised birthweight centile were assessed using multinomial logistic regression (with AGA as the reference category) to allow for non-linear relationships between maternal sleep and infant birth weight. Associations between each sleep measure and fetal distress were assessed using logistic regression. Analyses were carried out initially with minimal adjustment, including only infant gender as a covariable in order to increase precision. Subsequent analyses included adjustment for all potential confounders, including ethnicity. Since the relationship between sleep and depression is potentially bi-directional, we conducted a sensitivity analysis removing EPDS score from the confounder-adjusted model.

Our secondary aim was to examine potential ethnic differences in the associations between maternal sleep and infant outcomes. We conducted formal tests of interaction for differences in associations between Māori and non-Māori women, and present the results of these tests and all confounder-adjusted stratified analyses in online supplementary material. As an illustration of the general pattern of ethnic differences in associations, where the point estimate suggested a strong association $(\mathrm{OR} \geq 2)$ in either ethnicity, we present ethnic stratified results in the main paper as a graph. We chose this approach rather than using a $\mathrm{p}$ value threshold because of low statistical power, particularly in Māori women. We used the maximum available sample size for each separate sleep measure in our ethnic stratified analyses, rather than restrict analyses to women with complete data on all sleep measures.

\section{RESULTS}

\section{Sample definition}

Complete data on sleep in late pregnancy, birth weight, fetal distress and potential confounders were available for 633 women; 194 Māori women and 439 non-Māori women (46\% and $57 \%$ of the original cohort). Compared with those excluded due to missing data, women included in our analysis tended to be slightly older, live in areas of lower deprivation, were less likely to identify as Māori, and more likely nulliparous (see online supplementary table S1). A detailed breakdown of the missing data for each variable is presented in online supplementary table S2. Some differences in pre-pregnancy sleep were noted, with women included in our analysis less likely to sleep for nine or more hours per night (see online supplementary table S1). Differences in sleep during late pregnancy were less evident, although there was some evidence that women included in our analysis were more likely to have poor quality sleep as measured by the GSDS and less likely to sleep for less than 6 or 9 or more hours per night compared with those excluded (see online supplementary table S3).

\section{Participant characteristics}

Women who identified as Māori were more likely to be younger, smoke, live in more deprived areas (higher score on NZDep), have higher parity and higher EPDS depression scores. The prevalence of SGA and LGA was broadly similar in Māori and non-Māori women, but $13.9 \%$ of infants born to Māori women experienced fetal distress compared with $3.2 \%$ of infants born to non-Māori women (table 1).

\section{Maternal sleep parameters}

Women who identified as Māori were more likely to report a short $(\leq 6 \mathrm{~h})$ or long $(\geq 9 \mathrm{~h})$ pre-pregnancy sleep duration compared with non-Māori women (table 1). Ethnic differences in late pregnancy sleep were also evident, including a higher prevalence in Māori women of daytime sleepiness; sleep disturbance due to back, neck or joint pain, feeling too hot or cold, not being able to get comfortable, or not being able to sleep; long sleep duration; and leg twitching (table 2). 
Table 1 Participant characteristics by ethnicity

\begin{tabular}{|c|c|c|c|c|}
\hline & Statistic & $\begin{array}{l}\text { Mother identifies } \\
\text { as Māori }(\mathrm{N}=194)\end{array}$ & $\begin{array}{l}\text { Mother identifies } \\
\text { as non-Māori }(\mathrm{N}=439)\end{array}$ & p Value ${ }^{*}$ \\
\hline Maternal age (years) & Mean (SD) & $29.4(6.1)$ & $32.8(4.8)$ & $<0.001$ \\
\hline NZ Deprivation Index (tenths) & Median (IQR) & 7 (4 to 9$)$ & 4 (2 to 6$)$ & $<0.001$ \\
\hline Parity & Median (IQR) & 1 (0 to 2$)$ & $0(0$ to 1$)$ & 0.003 \\
\hline Gestational age at delivery (weeks) & Median (IQR) & 40 (39 to 41$)$ & 40 (39 to 41$)$ & 0.59 \\
\hline Maternal BMI $\left(\mathrm{kg} / \mathrm{m}^{2}\right)$ & Median (IQR) & $27.4(23.0$ to 30.8$)$ & 23.7 (21.5 to 26.8$)$ & $<0.001$ \\
\hline EPDS score in late pregnancy & Median (IQR) & 8 (5 to 12$)$ & $7(4$ to 11$)$ & 0.04 \\
\hline Maternal smoking in late pregnancy & $\mathrm{N}(\%)$ & $30(15.5 \%)$ & $12(2.7 \%)$ & $<0.001$ \\
\hline \multicolumn{5}{|l|}{ Sleep duration before pregnancy } \\
\hline Short sleep ( $\leq 6 \mathrm{~h})$ & $\mathrm{N}(\%)$ & $11(5.7 \%)$ & $12(2.7 \%)$ & \\
\hline Average sleep (>6 to <9 h) & & $114(58.8 \%)$ & $341(77.7 \%)$ & \\
\hline Long sleep $(\geq 9 \mathrm{~h})$ & & $69(35.6 \%)$ & $86(19.6 \%)$ & $<0.001$ \\
\hline 3 or more poor nights' sleep before pregnancy & $\mathrm{N}(\%)$ & $22(11.3 \%)$ & $38(8.7 \%)$ & 0.29 \\
\hline Birth weight & $\mathrm{N}(\%)$ & & & \\
\hline Small for gestational age & & $13(6.7 \%)$ & $43(9.8 \%)$ & \\
\hline Appropriate for gestational age & & $149(76.8 \%)$ & $341(77.7 \%)$ & \\
\hline Large for gestational age & & $32(16.5 \%)$ & $55(12.5 \%)$ & 0.22 \\
\hline Fetal distress & $\mathrm{N}(\%)$ & 27 (13.9\%) & $14(3.2 \%)$ & $<0.001$ \\
\hline
\end{tabular}

${ }^{*}$ Derived from linear regression when comparing means, non-parametric test of equality of medians, or $\chi^{2}$ tests for categorical variables. BMI, body mass index; EPDS, Edinburgh Postnatal Depression Scale; NZ, New Zealand.

Poor sleep quality was common in all women in late pregnancy, with $84 \%$ of Māori and $83 \%$ of non-Māori women reporting three or more nights of poor quality sleep in late pregnancy (table 2) compared with $11.3 \%$ and $8.7 \%$ retrospective pre-pregnancy reports (table 1 ). All results were similar with and without adjustment for EPDS score, so only results with this variable included in the set of confounders are presented.

\section{Maternal sleep in relation to SGA infants}

In analysis of Māori and non-Māori women combined, there was no strong evidence that poor sleep quality or other sleep parameters were associated with SGA (see online supplementary table S4 for minimally adjusted results and table 3 for confounder-adjusted results). Long sleep duration $(\geq 9 \mathrm{~h})$ was weakly associated with SGA (OR 1.6 after adjustment for confounders, with 95\% CI 0.8 to $3.2, \mathrm{p}=0.19)$. Among reasons for sleep disturbance, only nasal congestion showed a suggestion of association with SGA (OR 1.8 with $95 \%$ CI 1.0 to $3.2, \mathrm{p}=0.06$ ). Snoring, pregnancy-onset snoring, chronic snoring, leg twitching, pregnancy-onset leg twitching and restless legs showed no association with SGA. There was a weak association between breathing pauses and SGA (OR 2.8 with $95 \%$ CI 0.9 to $9.0, \mathrm{p}=0.08$ ), but this association was not observed for pregnancy-onset breathing pauses.

\section{Maternal sleep in relation to LGA infants}

There was a strong association between pregnancy-onset breathing pauses and LGA (OR 3.5 with 95\% CI 1.3 to 9.6, $\mathrm{p}=0.01)$. Other maternal sleep measures were either not associated with LGA or only weakly associated (see online supplementary table S4 for minimally adjusted results and table 3 for confounder-adjusted results).

\section{Maternal sleep in relation to fetal distress}

There was no evidence of associations between sleep quality, daytime sleepiness, sleep duration or changes in sleep duration and fetal distress (table 4) for both the pre-pregnancy and late pregnancy reports. Sleep disturbance due to feeling too hot or too cold had a higher odds of fetal distress (OR 1.7 with $95 \%$ CI 0.9 to 3.6, $\mathrm{p}=0.13$ ) but did not reach statistical significance. Pregnancy-onset leg twitching was associated with a threefold higher odds of fetal distress (OR 3.3 with $95 \%$ CI 1.1 to $10.0, \mathrm{p}=0.03$ ).

\section{Ethnic differences in the associations between maternal sleep and infant outcomes}

There was statistical evidence of Māori/non-Māori differences in associations between sleep in late pregnancy and birth outcomes for a small number of sleep measures (see online supplementary tables S5-7). Considering all sleep measures, regardless of whether the interaction test provided statistical evidence for ethnic differences, the general pattern was for associations to be stronger in Māori women (figures 1 and 2). For example, the OR for fetal distress due to feeling too hot or too cold was 4.9 in Māori women (95\% CI 1.6 to $15.0, \mathrm{p}<0.001)$ compared with 0.5 in non-Māori women (95\% CI 0.2 to 1.6 ) and the OR for fetal distress due to restless legs was 2.6 in Māori women (95\% CI 0.9 to 7.2 , $\mathrm{p}=0.07)$ compared with 0.3 in non-Māori women $(95 \%$ CI 0.0 to 2.2, $\mathrm{p}=0.22$ ).

\section{DISCUSSION}

In this cohort of women in the last trimester of pregnancy, we observed that self-reported poor sleep quality and sleep disturbances were common, with $84 \%$ of 
Table 2 Sleep in late pregnancy by ethnicity

\section{Mother identifies \\ as Māori $(\mathrm{N}=194)$}

\begin{abstract}
Poor sleep quality
3 or more nights poor sleep

GSDS total

GSDS quality subscale
\end{abstract}

GSDS daytime sleepiness subscale

Sleep disturbances on three or more nights

Going to the bathroom

Pain in back/neck/joints

Dreams

Nightmares

Heartburn

Nasal congestion

Leg cramps

Contractions

Feeling too hot or too cold

Thinking or worrying about things

Baby moving about

Other children

Just cannot get comfortable

Just cannot get to sleep

Disturbed by partner

Sleep duration

Short sleep $(\leq 6 \mathrm{~h})$

Average sleep ( $>6$ to $<9 \mathrm{~h}$ )

Long sleep $(\geq 9 \mathrm{~h})$

Change in sleep duration between pre-pregnancy and late pregnancy*

Increased

No change

Decreased

Sleep disorders

Snoring

Chronic snoring

Pregnancy-onset snoring

Breathing pauses

Pregnancy-onset breathing pauses

Leg twitching

Pregnancy-onset leg twitching

Restless legs

Excessive daytime sleepiness ( $E S S \geq 10$ )
$163(84.0 \%)$

$167(86.1 \%)$

$165(85.1 \%)$

$112(57.7 \%)$

$177(91.2 \%)$

$147(75.8 \%)$

$83(42.8 \%)$

$21(10.8 \%)$

$72(37.1 \%)$

$50(25.8 \%)$

$62(32.0 \%)$

$31(16.0 \%)$

$112(57.7 \%)$

$95(49.0 \%)$

$127(65.5 \%)$

$60(30.9 \%)$

$149(76.8 \%)$

$103(53.1 \%)$

$50(25.8 \%)$

57 (29.4\%)

$90(46.4 \%)$

$47(24.2 \%)$

$28(14.4 \%)$

$83(42.8 \%)$

$83(42.8 \%)$

$47(24.2 \%)$

$21(10.8 \%)$

$22(11.3 \%)$

$11(5.7 \%)$

$8(4.1 \%)$

$36(18.6 \%)$

$9(4.6 \%)$

$28(14.4 \%)$

60 (30.9\%)
Mother identifies

as non-Māori $(\mathrm{N}=439)$

$p$ Value for comparison

$364(82.9 \%)$

0.73

$380(86.6 \%)$

$366(83.4 \%)$

202 (46.0\%)

0.87

0.60

0.01

396 (90.2\%)

$269(61.3 \%)$

$139(31.7 \%)$

$29(6.6 \%)$

$162(36.9 \%)$

$118(26.9 \%)$

$108(24.6 \%)$

$42(9.6 \%)$

$204(46.5 \%)$

191 (43.5\%)

$241(54.9 \%)$

$101(23.0 \%)$

$271(61.7 \%)$

$166(37.8 \%)$

$98(22.3 \%)$

0.68

$<0.001$

0.01

0.07

0.77

0.77

0.05

0.02

0.01

0.20

0.01

0.04

$<0.001$

$<0.001$

0.34

$118(26.9 \%)$

$247(56.3 \%)$

$74(16.9 \%)$

0.04

$62(14.1 \%)$

$210(47.8 \%)$

167 (38.0\%)

0.47

$104(23.7 \%)$

0.88

$29(6.6 \%)$

$82(18.7 \%)$

0.07

$13(3.0 \%)$

$12(2.7 \%)$

32 (7.3\%)

26 (5.9\%)

$81(18.5 \%)$

$96(21.9 \%)$

Values are number (percent); $p$ values are obtained from $\chi^{2}$ tests.

*Defined as movement between the defined categories of short sleep ( $\leq 6 \mathrm{~h})$, average sleep ( $>6$ and $<9 \mathrm{~h})$ and long sleep ( $\geq 9 \mathrm{~h})$.

ESS, Epworth Sleepiness Scale; GSDS, General Sleep Disturbance Scale.

Māori women and $83 \%$ of non-Māori women reporting three or more nights of poor quality sleep per week. Many women experienced reductions in sleep duration and quality from pre-pregnancy to $35-37$ weeks gestation, and problematic sleep tended to be more common in women identifying as Māori.

\section{Maternal sleep in relation to infant birthweight and fetal distress}

This study is the first to report associations between a large range of maternal sleep measures and infant outcomes. We found little evidence of associations between most of the sleep measures we investigated and the risk of a baby being born SLA or LGA. There was weak evidence that experiencing breathing pauses on three or more nights per week in late pregnancy (a measure of sleep apnoea) was associated with higher risk of both SGA and LGA, which was robust to adjustment for selfreported maternal BMI. For SGA, this association was not present for pregnancy-onset breathing pauses (ie, women who experience this symptom in late pregnancy but did not experience it pre-pregnancy). For LGA, the association was observed for both current breathing pauses and pregnancy-onset breathing pauses, but was strongest for pregnancy-onset. Our results provide some evidence that pregnancy-onset leg twitching and frequent sleep disturbance due to feeling too hot or too cold are associated with higher risk of fetal distress. The 
Table 3 Confounder-adjusted associations between sleep in late pregnancy and customised infant birthweight centile $(\mathrm{N}=633)$

\begin{tabular}{|c|c|c|c|}
\hline \multirow{2}{*}{ Measure of sleep } & \multicolumn{3}{|c|}{ Birth size using customised birthweight centiles for the NZ population } \\
\hline & $\begin{array}{l}\text { Small for gestational } \\
\text { age }(<10 \text { th centile) } \\
\mathrm{N}=56\end{array}$ & $\begin{array}{l}\text { Appropriate for } \\
\text { gestational age } \\
\mathrm{N}=490\end{array}$ & $\begin{array}{l}\text { Large for gestational } \\
\text { age (>90th centile) } \\
\mathrm{N}=87\end{array}$ \\
\hline \multicolumn{4}{|l|}{ Poor sleep quality } \\
\hline 3 or more nights poor sleep & $1.0(0.4$ to 2.0$) p=0.89$ & 1 (ref) & $0.9(0.5$ to 1.6$) p=0.61$ \\
\hline GSDS total & $1.1(0.5$ to 2.7$) p=0.77$ & 1 (ref) & $1.0(0.5$ to 2.0$) p=0.99$ \\
\hline GSDS quality subscale & $1.0(0.4$ to 2.1$) p=0.90$ & 1 (ref) & $0.7(0.4$ to 1.3$) p=0.23$ \\
\hline GSDS daytime sleepiness subscale & $1.3(0.7$ to 2.3$) p=0.45$ & 1 (ref) & $1.2(0.7$ to 1.9$) \mathrm{p}=0.52$ \\
\hline \multicolumn{4}{|c|}{ Sleep disturbances on three or more nights per week } \\
\hline Going to the bathroom & $1.1(0.4$ to 3.0$) p=0.82$ & 1 (ref) & $1.5(0.6$ to 3.6$) p=0.39$ \\
\hline Pain in back/neck/joints & $1.1(0.6$ to 2.0$) p=0.81$ & 1 (ref) & $1.0(0.6$ to 1.6$) p=0.87$ \\
\hline Dreams & $0.8(0.4$ to 1.5$) p=0.46$ & 1 (ref) & $1.5(0.9$ to 2.4$) p=0.10$ \\
\hline Nightmares & $0.7(0.2$ to 2.4$) p=0.57$ & 1 (ref) & $0.5(0.2$ to 1.5$) p=0.21$ \\
\hline Heartburn & $0.6(0.3$ to 1.1$) p=0.09$ & 1 (ref) & $0.8(0.5$ to 1.3$) p=0.29$ \\
\hline Nasal congestion & $1.8(1.0$ to 3.2$) p=0.06$ & 1 (ref) & $1.0(0.6$ to 1.7$) p=0.95$ \\
\hline Leg cramps & $0.6(0.3$ to 1.2$) p=0.14$ & 1 (ref) & $1.2(0.7$ to 2.0$) p=0.55$ \\
\hline Contractions & $1.0(0.4$ to 2.6$) p=0.96$ & 1 (ref) & $1.3(0.6$ to 2.6$) p=0.48$ \\
\hline Feeling too hot or too cold & $0.9(0.5$ to 1.6$) p=0.81$ & 1 (ref) & $1.2(0.8$ to 2.0$) p=0.40$ \\
\hline Thinking or worrying about things & $1.1(0.6$ to 1.9$) \mathrm{p}=0.85$ & 1 (ref) & $1.6(1.0$ to 2.6$) p=0.08$ \\
\hline Baby moving about & $1.3(0.7$ to 2.4$) p=0.34$ & 1 (ref) & $1.3(0.8$ to 2.1$) p=0.29$ \\
\hline Other children & $1.1(0.5$ to 2.3$) p=0.76$ & 1 (ref) & $1.1(0.6$ to 2.1$) p=0.79$ \\
\hline Just cannot get comfortable & $1.2(0.7$ to 2.3$) p=0.48$ & 1 (ref) & $1.2(0.7$ to 2.0$) p=0.53$ \\
\hline Just cannot get to sleep & $0.7(0.4$ to 1.3$) p=0.27$ & 1 (ref) & $1.4(0.9$ to 2.2$) p=0.19$ \\
\hline Disturbed by partner & $0.2(0.1$ to 0.7$) p=0.01$ & 1 (ref) & $1.4(0.8$ to 2.3$) p=0.26$ \\
\hline \multicolumn{4}{|l|}{ Sleep duration } \\
\hline Short sleep $(\leq 6 \mathrm{~h})$ & $0.9(0.4$ to 1.8$) \mathrm{p}=0.72$ & 1 (ref) & $1.1(0.6$ to 1.9$) p=0.76$ \\
\hline Average sleep $(>6$ to $<9 \mathrm{~h}$ ) & 1 (ref) & 1 (ref) & 1 (ref) \\
\hline Long sleep ( $\geq 9 \mathrm{~h}$ ) & $1.6(0.8$ to 3.2$) p=0.19$ & 1 (ref) & $1.1(0.6$ to 2.0$) p=0.83$ \\
\hline \multicolumn{4}{|c|}{ Change in sleep duration between pre-pregnancy and late pregnancy ${ }^{*}$} \\
\hline Increased & $1.4(0.6$ to 3.2$) p=0.47$ & 1 (ref) & $1.4(0.7$ to 2.9$) p=0.29$ \\
\hline No change & 1 (ref) & 1 (ref) & 1 (ref) \\
\hline Decreased & $0.8(0.4$ to 1.5$) p=0.50$ & 1 (ref) & $1.3(0.7$ to 2.2$) p=0.37$ \\
\hline \multicolumn{4}{|l|}{ Sleep disorders } \\
\hline Snoring & $1.2(0.6$ to 2.4$) p=0.52$ & 1 (ref) & $1.2(0.7$ to 2.0$) p=0.58$ \\
\hline Chronic snoring & $1.6(0.6$ to 4.0$) p=0.32$ & 1 (ref) & $1.7(0.8$ to 3.7$) p=0.14$ \\
\hline Pregnancy-onset snoring & $0.8(0.4$ to 1.8$) p=0.58$ & 1 (ref) & $1.1(0.6$ to 2.1$) p=0.75$ \\
\hline Breathing pauses & $2.8(0.9$ to 9.0$) p=0.08$ & 1 (ref) & $2.0(0.7$ to 5.7$) p=0.21$ \\
\hline Pregnancy-onset breathing pauses & $1.7(0.4$ to 7.9$) \mathrm{p}=0.52$ & 1 (ref) & $3.5(1.3$ to 9.6$) p=0.01$ \\
\hline Les twitching & $1.4(0.6$ to 3.5$) p=0.45$ & 1 (ref) & $1.0(0.5$ to 2.2$) \mathrm{p}=0.99$ \\
\hline Pregnancy-onset leg twitching & $0.2(0.0$ to 1.9$) \mathrm{p}=0.18$ & 1 (ref) & $0.6(0.2$ to 2.1$) p=0.42$ \\
\hline Restless legs & $0.9(0.4$ to 2.0$) p=0.86$ & 1 (ref) & $1.5(0.8$ to 2.6$) p=0.19$ \\
\hline Excessive daytime sleepiness ( $E S S \geq 10$ ) & $0.8(0.4$ to 1.6$) p=0.52$ & 1 (ref) & $1.4(0.8$ to 2.4$) p=0.19$ \\
\hline
\end{tabular}

Coefficients are ORs with 95\% Cls from logistic regressions, adjusted for infant gender, ethnicity (Māori or non-Māori), NZ Deprivation Index, maternal age, parity, BMI, EPDS score, smoking (any vs none), pre-pregnancy sleep duration and number of poor nights' sleep.

${ }^{*}$ Defined as movement between categories of short sleep $(\leq 6 \mathrm{~h})$, average sleep ( $>6$ and $<9 \mathrm{~h}$ ) and long sleep $(\geq 9 \mathrm{~h})$.

BMI, body mass index; EPDS, Edinburgh Postnatal Depression Scale; ESS, Epworth Sleepiness Scale; GSDS, General Sleep Disturbance Scale; NZ, New Zealand.

association between breathing pauses and birth size, particularly LGA, could to some degree reflect gestational diabetes due to known associations between obstructive sleep apnoea (OSA) and glucose intolerance and between gestational diabetes and macrosomia. In this study, 5\% ( $\mathrm{N}=5)$ of mothers with LGA infants reported having diabetes, compared with $2 \%(\mathrm{~N}=15)$ of mothers with AGA infants. Owing to the small numbers, it was not possible to further evaluate the role of diabetes in the associations we observe.
Several other studies have examined sleep in pregnancy in relation to infant birth weight. Snoring has been postulated as a determinant of fetal growth and hence infant birth weight. A prospective cohort study of 1673 women in the USA found that chronic snoring (snoring both before and during pregnancy) was associated with a higher risk of the infant being SGA (OR $1.65,95 \%$ CI 1.02 to 2.66 ), but there was no association between snoring and LGA. ${ }^{31}$ Among 1091 women in a prospective cohort in Crete, Greece, severe snoring 
Table 4 Associations between sleep in late pregnancy and fetal distress $(\mathrm{N}=633)$

\begin{tabular}{|c|c|c|}
\hline \multirow[t]{2}{*}{ Measure of sleep } & \multicolumn{2}{|l|}{ OR and $95 \% \mathrm{Cl}$ for fetal distress } \\
\hline & Minimally adjusted association* & Adjusted association $\dagger$ \\
\hline \multicolumn{3}{|l|}{ Poor sleep quality } \\
\hline 3 or more nights poor sleep & $0.8(0.4$ to 1.9$) p=0.65$ & 0.8 (0.3 to 1.9$) p=0.61$ \\
\hline GSDS total & $0.7(0.3$ to 1.7$) p=0.48$ & 0.7 (0.3 to 1.8$) p=0.45$ \\
\hline GSDS quality subscale & $1.4(0.5$ to 3.7$) p=0.50$ & $1.4(0.5$ to 3.9$) p=0.52$ \\
\hline GSDS daytime sleepiness subscale & $1.3(0.7$ to 2.5$) p=0.38$ & 1.0 (0.5 to 2.2) $p=0.92$ \\
\hline \multicolumn{3}{|c|}{ Sleep disturbances on three or more nights per week } \\
\hline Going to the bathroom & $0.6(0.2$ to 1.4$) p=0.22$ & $0.6(0.2$ to 1.5$) p=0.25$ \\
\hline Pain in back/neck/joints & $1.4(0.7$ to 2.9$) p=0.31$ & $1.0(0.5$ to 2.2$) p=0.93$ \\
\hline Dreams & $1.5(0.8$ to 2.8$) p=0.24$ & $1.2(0.6$ to 2.4$) p=0.62$ \\
\hline Nightmares & $1.3(0.4$ to 3.8$) p=0.64$ & 0.9 (0.3 to 2.9$) p=0.87$ \\
\hline Heartburn & $1.0(0.5$ to 1.9$) p=0.98$ & 1.1 (0.5 to 2.1$) p=0.88$ \\
\hline Nasal congestion & $1.0(0.5$ to 2.1$) p=0.98$ & $0.9(0.4$ to 2.0$) p=0.86$ \\
\hline Leg cramps & $0.7(0.3$ to 1.6$) p=0.42$ & $0.6(0.2$ to 1.3$) p=0.17$ \\
\hline Contractions & $0.4(0.1$ to 1.6$) p=0.19$ & $0.3(0.1$ to 1.4$) p=0.14$ \\
\hline Feeling too hot or too cold & 2.0 (1.0 to 3.9$) p=0.04$ & 1.7 (0.9 to 3.6$) p=0.13$ \\
\hline Thinking or worrying about things & $1.2(0.6$ to 2.2$) p=0.66$ & $1.0(0.5$ to 2.0$) p=0.90$ \\
\hline Baby moving about & $1.1(0.6$ to 2.1$) p=0.76$ & $1.0(0.5$ to 2.1$) p=0.96$ \\
\hline Other children & $0.7(0.3$ to 1.5$) p=0.37$ & $1.4(0.5$ to 3.7$) p=0.56$ \\
\hline Just cannot get comfortable & $0.7(0.4$ to 1.3$) p=0.29$ & $0.5(0.2$ to 1.0$) p=0.04$ \\
\hline Just cannot get to sleep & $1.2(0.6$ to 2.2$) p=0.62$ & $0.9(0.4$ to 1.8$) p=0.68$ \\
\hline Disturbed by partner & $0.9(0.4$ to 2.0$) p=0.85$ & $0.8(0.4$ to 1.8$) p=0.63$ \\
\hline Sleep duration & 1 (ref) & 1 (ref) \\
\hline Short sleep ( $\leq 6 \mathrm{~h})$ & $1.4(0.7$ to 2.8$) p=0.42$ & $1.6(0.7$ to 3.5$) p=0.26$ \\
\hline Average sleep (>6 to $<9 \mathrm{~h}$ ) & 1 (ref) & 1 (ref) \\
\hline Long sleep ( $\geq 9 \mathrm{~h})$ & $1.4(0.6$ to 3.1$) p=0.47$ & $1.2(0.5$ to 3.0$) p=0.66$ \\
\hline \multicolumn{3}{|c|}{ Change in sleep duration between pre-pregnancy and late pregnancy } \\
\hline Increased & $1.4(0.5$ to 3.9$) p=0.47$ & $1.4(0.5$ to 4.1$) p=0.55$ \\
\hline No change & 1 (ref) & 1 (ref) \\
\hline Decreased & $1.8(0.9$ to 3.7$) p=0.09$ & $1.5(0.7$ to 3.4$) p=0.28$ \\
\hline Sleep disorders & 1 (ref) & 1 (ref) \\
\hline Snoring & $1.2(0.6$ to 2.4$) p=0.65$ & $1.1(0.5$ to 2.4$) p=0.79$ \\
\hline Chronic snoring & $1.7(0.6$ to 4.5$) p=0.30$ & 1.5 (0.5 to 4.2$) p=0.48$ \\
\hline Pregnancy-onset snoring & $0.2(0.1$ to 1.0$) p=0.06$ & $0.3(0.1$ to 1.2$) p=0.09$ \\
\hline Breathing pauses & $0.6(0.1$ to 4.8$) p=0.65$ & $0.5(0.1$ to 4.0$) p=0.50$ \\
\hline Pregnancy-onset breathing pauses & $0.8(0.1$ to 6.0$) p=0.81$ & $0.8(0.1$ to 6.7$) p=0.86$ \\
\hline Leg twitching & $1.1(0.4$ to 3.0$) p=0.79$ & $0.5(0.2$ to 1.6$) p=0.28$ \\
\hline Pregnancy-onset leg twitching & $2.6(0.9$ to 7.0$) p=0.07$ & $3.3(1.1$ to 10.0$) p=0.03$ \\
\hline Restless legs & $1.2(0.5$ to 2.6$) p=0.71$ & $1.1(0.5$ to 2.5$) p=0.89$ \\
\hline Excessive daytime sleepiness (ESS $\geq 10$ ) & $1.1(0.5$ to 2.3$) p=0.75$ & 1.0 (0.5 to 2.2$) p=0.93$ \\
\hline \multicolumn{3}{|c|}{$\begin{array}{l}\text { Coefficients are ORs and } 95 \% \text { Cls for fetal distress }(\mathrm{n}=41) \text { compared with no fetal distress. } \\
\text { ^Adjusted for infant gender. } \\
\text { †Adjusted for infant gender, ethnicity (Māori or non-Māori), NZ Deprivation Index, maternal age, parity, BMI, EPDS score, gestational age at } \\
\text { delivery, smoking (any vs none), pre-pregnancy sleep duration and number of poor nights' sleep. } \\
\text { fDefined as movement between the defined categories of short sleep }(\leq 6 \mathrm{~h}) \text {, average sleep ( }>6 \text { and }<9 \mathrm{~h}) \text { and long sleep ( } \geq 9 \mathrm{~h}) \text {. } \\
\text { BMI, body mass index; EPDS, Edinburgh Postnatal Depression Scale; ESS, Epworth Sleepiness Scale; GSDS, General Sleep Disturbance } \\
\text { Scale; NZ, New Zealand. }\end{array}$} \\
\hline
\end{tabular}

(women who reported snoring frequently or always) was associated with higher risk of low birth weight $(<2.5 \mathrm{~kg})$ and with customised birth weight below the 10th centile; relative risks after adjusting for various confounders (including maternal BMI) were 2.6 (95\% CI 1.2 to 5.4 ) and 2.0 (1.0 to 3.8$).{ }^{29}$ We did not replicate these findings in our analyses; we found no evidence of an association between snoring (current snoring on three or more nights per week, pregnancy-onset snoring on three or more nights per week, or chronic snoring defined as snoring on three or more nights per week both prepregnancy and in late pregnancy) and either SGA or LGA. The reasons for the difference between our results and those of previous studies are unclear. It is possible that our sample size was insufficient to detect the association, or that the lack of association in our cohort is due to population differences. Recruitment into the E Moe, Māmā cohort took place in late pregnancy (most women were between 35 and 37 weeks gestation at completion of the initial questionnaire); thus the number of 


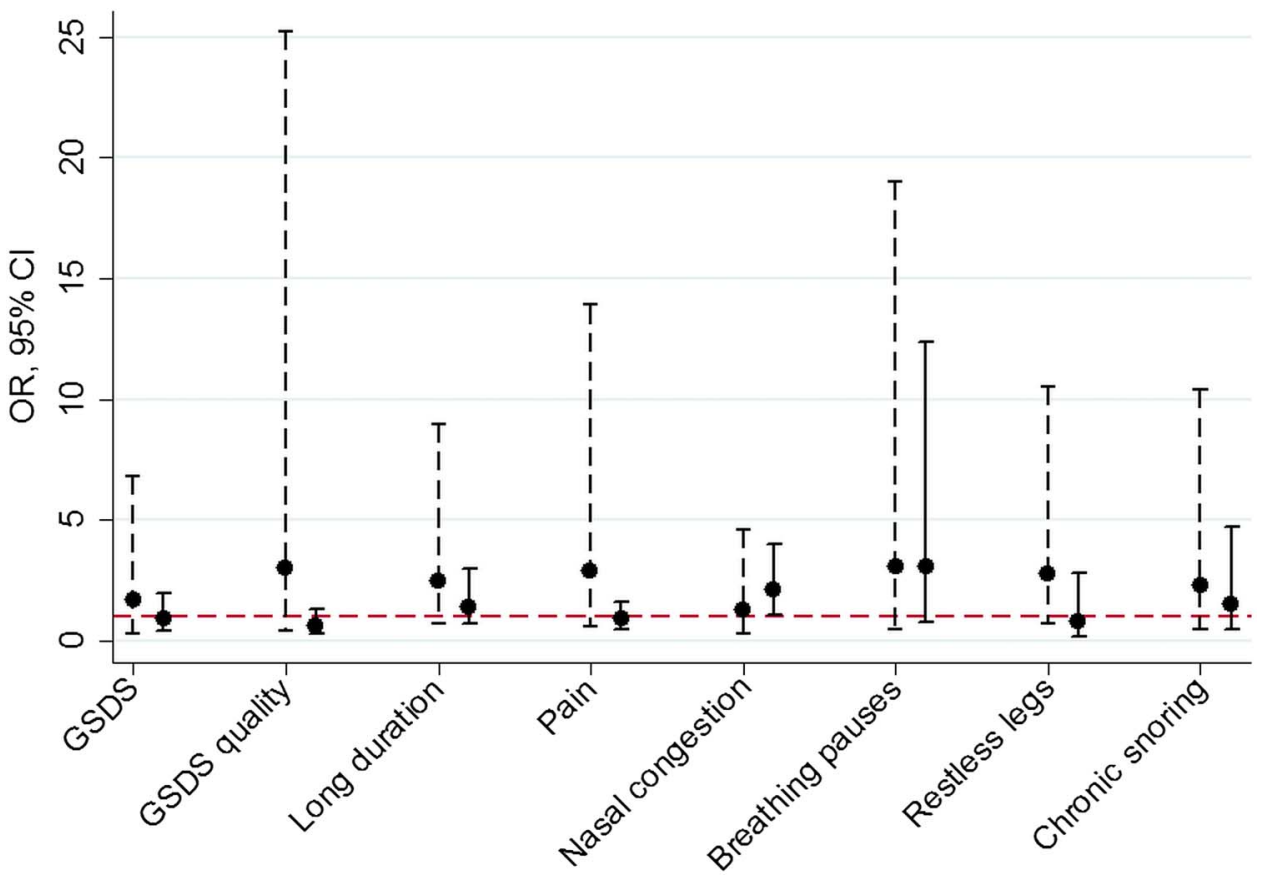

Figure 1 Associations between sleep in late pregnancy and small for gestational age stratified by mother's Māori versus non-Māori ethnicity. Legend: ORs and $95 \%$ Cls are shown for the association between measures of sleep in late pregnancy and small for gestational age (<10th customised birthweight centile) for Māori (dashed lines) and non-Māori (solid lines) women. The red dashed line indicates the null value $(O R=1)$. Associations are shown for sleep measures where the $O R \geq 2$ in either ethnicity. GSDS is the General Sleep Disturbance Scale; GSDS quality is the sleep quality subscale of the GSDS; long duration is sleep duration $\geq 9 \mathrm{~h}$; pain refers to disturbed sleep on three or more nights per week due to back, neck or shoulder pain; nasal congestion refers to disturbed sleep on three or more nights per week due to nasal congestion; breathing pauses refers to breathing pauses on three or more nights per week; restless legs is defined as having an urge to move legs (usually accompanied by unpleasant sensations) that is worse at night, more noticeable when resting and relieved by movement; chronic snoring is snoring on three or more nights a week both pre-pregnancy and in late pregnancy.

preterm births in our sample is extremely small. Other studies included a higher percentage of preterm infants, and this may explain some of the differences in results. Definitions of snoring also differ slightly between studies.

In addition to the studies on snoring and birth weight, a small study involving 14 pregnant women with OSA and 27 controls found that OSA was associated with a customised birth weight less than the 10th centile or a reduction in customised centile of more than $33 \%$ between the 32-week ultrasound and delivery. ${ }^{47}$ Our analysis, which included a much larger number of women but from a population-based sample, rather than clinical cases, found weak evidence of association between frequent (3 or more nights per week) breathing pauses during sleep and neither SGA nor LGA. Given the wide CIs in our analysis, this finding requires replication in other large population-based studies, and examination in studies with objective sleep measures, which may shed light on the mechanisms.

We found no evidence that sleep duration was associated with SGA or LGA. A previous study of 200 women found that sleep duration was only weakly related to birth weight, with mean birth weight $3516.9 \mathrm{~g}$ in women who slept more than $8 \mathrm{~h}$ in the third trimester, $3502.2 \mathrm{~g}$ in those who slept $6-8 \mathrm{~h}$ and $3442.4 \mathrm{~g}$ in those who slept less than $6 \mathrm{~h}^{48}$ This study, however, considered birth weight as a continuum, and so is not directly comparable to our analysis of SGA and LGA.

Fetal distress has, to our knowledge, not been considered as a potential consequence of poor sleep in pregnancy. It is possible that our observed associations with leg twitching and sleep disturbance due to feeling too hot or too cold may be due to underlying medical conditions, although we could find no clear evidence of this using the available (self-reported, free-text) medical data. Since this is the first study to examine the associations between sleep in pregnancy and fetal distress, the observed associations with leg twitching and sleep disturbance due to feeling too hot or cold require confirmation in other large studies.

\section{Ethnic differences in the associations between maternal sleep and infant outcomes}

Stark inequities in health exist between the Māori and non-Māori populations of NZ, for both sleep ${ }^{49} 50$ and infant $^{32}$ outcomes, and it is thus imperative to examine ethnic differences in health research. The women identifying as Māori in this study reported worse sleep during pregnancy than the non-Māori women, for example, 


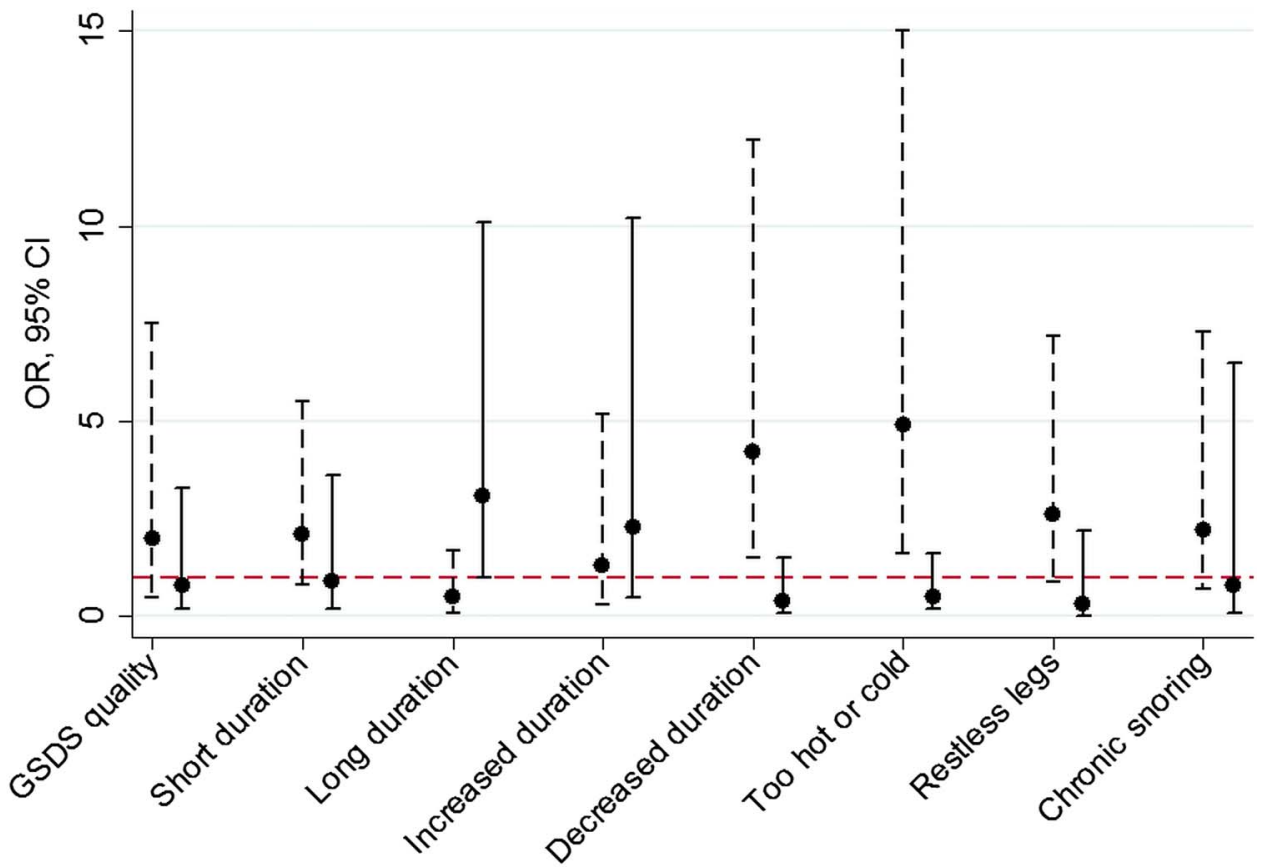

Figure 2 Associations between sleep in late pregnancy and fetal distress stratified by mother's Māori versus non-Māori ethnicity. Legend: ORs and 95\% Cls are shown for the association between measures of sleep in late pregnancy and fetal distress for Māori (dashed lines) and non-Māori (solid lines) women. Associations are shown for sleep measures where the $\mathrm{OR} \geq 2$ in either ethnicity. GSDS quality is the quality subscale of the General Sleep Disturbance Scale; short duration is sleep duration $\leq 6 \mathrm{~h}$; long duration is sleep duration $\geq 9 \mathrm{~h}$; increased duration means moving from short $(\leq 6 \mathrm{~h})$ to average $(>6$ to $<9 \mathrm{~h})$ or average to long $(9 \geq \mathrm{h})$ duration of sleep from pre-pregnancy to late pregnancy; decreased duration is the reverse of this; too hot or cold is sleep disturbance on three or more nights per week due to feeling too hot or too cold; restless legs is defined as having an urge to move legs (usually accompanied by unpleasant sensations) that is worse at night, more noticeable when resting and relieved by movement; chronic snoring is snoring on three or more nights a week both pre-pregnancy and in late pregnancy.

having a higher prevalence of short sleep duration and many sleep disturbances. The reasons for this difference are unclear and require further exploration, for example, in qualitative studies. For many of the associations we examined, the associations of sleep during pregnancy with birthweight or fetal distress were either stronger in women identifying as Māori, or present only in Māori women. For example, global GSDS scores; the GSDS quality subscale; long sleep duration; sleep disturbance due to back, neck or joint pain; and restless legs demonstrated stronger associations with SGA in Māori women, and the GSDS quality subscale, short sleep duration, decreased sleep duration from prepregnancy to late pregnancy, sleep disturbance due to feeling too hot or too cold, and restless legs demonstrated stronger associations with fetal distress in Māori women. It therefore appears that for many of the associations we see in the combined population, these are being driven by the Māori women.

The reasons underlying the stronger associations between maternal sleep and infant outcomes in Māori women are uncertain, but are likely to be complex and multifactorial. One possibility is that the self-reported sleep measures may capture different underlying processes for the Māori and non-Māori women (ie, women in these groups may interpret the questions slightly differently, or place different emphases on sleep as an aspect of health and well-being). The questionnaires used in this study have not been validated in a Māori population. A similar consideration is that some of the confounding variables we adjusted for may act differently in Māori and non-Māori women. A third potential explanation is that we have created binary variables indicating problematic/non-problematic sleep for each sleep measure, and it is possible that for each sleep measure, Māori women have on average worse levels than non-Māori women. Further research, including qualitative studies to explore potential differences in the interpretation of the sleep questionnaire and studies with objective measures of sleep, is required to disentangle these potential explanations. It is noteworthy that intervention studies designed to help with maternal sleep have shown greater improvement among more disadvantaged women. ${ }^{35}$

The finding of stronger associations between maternal sleep and infant outcomes emphasises an important need to focus on the sleep and other health concerns of Māori pregnant women in order to reduce inequalities in health for both the mother and her infant. ${ }^{44}{ }^{49} \mathrm{~A}$ multilevel approach to improve sleep may help all women, and address ethnic inequalities. This may involve better/more education for lead maternity carers and other health providers about the importance of sleep, the existence of ethnic inequalities, and how 
these might impact on mother and infant well-being; screening and identification of sleep problems in pregnancy; appropriate interventions/strategies for reducing and/or managing symptoms and monitoring the mother and infant; and policy-level interventions around funding and planning will be critical. Issues of ethnic inequalities in access to antenatal care are also likely to be important.

\section{Strengths and limitations}

In this analysis, we have utilised data from a large prospective cohort study with data on many potential confounding factors. A key strength of our approach is the availability of a broad array of measurements of sleep in late pregnancy, allowing us to test whether multiple facets of sleep have different consequences for birth outcomes. A consequence of this, however, is that we have conducted multiple statistical tests and there is a possibility that the associations we observe are false positives. Replication in other large cohort studies is essential to clarify the role of sleep parameters on birth outcomes, and where associations are replicated and confirmed, the underlying biological mechanisms need exploration in studies with objective sleep measures. The availability of data on pre-pregnancy and pregnancy snoring, leg twitching and breathing pauses enabled us to study differential consequences of pregnancy-onset sleep disorders, as emphasised by previous studies. ${ }^{31}$ Using self-reported maternal BMI means that there is a possibility of residual confounding due to measurement error in this variable. We were able to adjust for prepregnancy sleep duration and sleep quality in order to reduce confounding of our associations of interest, and to examine whether change in sleep duration from prepregnancy to late pregnancy was an important determinant of birth outcomes. However, it would also be interesting in future studies to examine the dynamic nature of sleep across the different stages of pregnancy. While our data on sleep in late pregnancy were self-reported and assessed at a single time point during late pregnancy, this is the case in most other studies on this topic. ${ }^{12} 31$ Future studies using objective measures of sleep such as actigraphy, particularly with repeated measures across different stages of pregnancy, will make a valuable contribution to this literature. We observed important differences in associations between Māori and non-Māori women. The non-Māori group represents a mixture of ethnicities, including other groups that suffer a health disadvantage in NZ, such as Pacific Island people. ${ }^{51} 52$ Thus, we may have underestimated the magnitude of Māori and non-Māori differences in the associations of interest, and we were unable to examine other ethnic differences due to small numbers in each group.

\section{CONCLUSION}

This analysis provides a novel angle to the emerging literature on the topic of sleep in pregnancy and birth complications, considering a broader range of sleep measures than previous studies, and evaluating ethnic inequalities in the associations between maternal sleep and infant outcomes. We did not replicate previous findings that snoring is associated with SGA, but our results tentatively suggest that breathing pauses (a measure of sleep apnoea) may be associated with both SGA and LGA, and that pregnancy-onset leg twitching and frequent sleep disturbance due to feeling too hot or too cold are associated with higher risk of fetal distress, particularly for women identifying as Māori. Initiatives to improve the sleep of pregnant women, and reduce ethnic inequalities, may improve infant outcomes.

Acknowledgements The authors would like to thank the various recruitment sites throughout New Zealand and Marnie Reinfelds, Monique McLeod, Dale Morgan and Irene Popata for their assistance with recruiting women. Thank you also to Ms Larine Sluggett, Ms Meg Thompson and Ms Perrine Boy for their help with data entry and preparation. The study received advice and support from: Dr Michel Sangalli, Dr Kristie Carter, Dr Kara Mihaere, Dr Cheryl Benn, Ms Bridget Robson and Ms Rosie Smith.

Contributors This study was conceived and designed by LDH, TLS, S-JP, BS and DM. TLS, S-JP, BS, MP, DM, KL, MH and PG had input in the design of the E Moe, Māmā study and data collection. LDH carried out statistical analysis and wrote the first draft of the manuscript. All authors contributed to critical revisions of the manuscript, interpretation of data and approved the final version.

Funding LDH is supported by a UK Medical Research Council Fellowship (G1002375). S-JP was the recipient of a Health Research Council of NZ Eru Pomare Fellowship in Maori Health. The study was funded by the Health Research Council of New Zealand, project grant (09/233). Gifts for participating women were provided by Simunovich Olive Estate.

Competing interests None declared.

Ethics approval Central Region Health and Disability Ethics Committee (protocol CEN 09/09/070).

Provenance and peer review Not commissioned; externally peer reviewed.

Data sharing statement No additional data are available.

Open Access This is an Open Access article distributed in accordance with the terms of the Creative Commons Attribution (CC BY 4.0) license, which permits others to distribute, remix, adapt and build upon this work, for commercial use, provided the original work is properly cited. See: http:// creativecommons.org/licenses/by/4.0/

\section{REFERENCES}

1. Odd D, Evans D, Emond A. Preterm birth, age at school entry and educational performance. PLOS ONE 2013;8:e76615.

2. Odd DE, Lingam R, Emond A, et al. Movement outcomes of infants born moderate and late preterm. Acta Paediatr 2013;102: 876-82.

3. Zammit S, Odd D, Horwood J, et al. Investigating whether adverse prenatal and perinatal events are associated with non-clinical psychotic symptoms at age 12years in the ALSPAC birth cohort. Psychol Med 2009;39:1457-67.

4. Kramer MS. The epidemiology of low birthweight. Nestle Nutr Inst Workshop Ser 2013;74:1-10.

5. McCowan LM, Thompson JM, Taylor RS, et al. Clinical prediction in early pregnancy of infants small for gestational age by customised birthweight centiles: findings from a healthy nulliparous cohort. PLOS ONE 2013;8:e70917.

6. Okun ML, Roberts JM, Marsland AL, et al. How disturbed sleep may be a risk factor for adverse pregnancy outcomes. Obstet Gynecol Surv 2009;64:273-80.

7. Baratte-Beebe KR, Lee KJ. Sources of mid-sleep awakenings in childbearing women. Clin Nurs Res 1999;8:386-97. 
8. Lee KA. Sleep during pregnancy and postpartum. In: Lee-Chiong T, ed. Encyclopedia of sleep medicine. John Wiley \& Sons, 2006:629-35.

9. Okun ML, Hall M, Coussons-Read ME. Sleep disturbances increase interleukin-6 production during pregnancy: implications for pregnancy complications. Reprod Sci 2007;14:560-7.

10. Okun ML, Kiewra K, Luther JF, et al. Sleep disturbances in depressed and nondepressed pregnant women. Depress Anxiety 2011;28:676-85.

11. Okun ML, Kline CE, Roberts JM, et al. Prevalence of sleep deficiency in early gestation and its associations with stress and depressive symptoms. J Womens Health (Larchmt) 2013;22:1028-37.

12. Okun ML, Luther JF, Wisniewski SR, et al. Disturbed sleep, a novel risk factor for preterm birth? J Womens Health (Larchmt) 2012;21:54-60.

13. Okun ML, Luther JF, Wisniewski SR, et al. Disturbed sleep and inflammatory cytokines in depressed and nondepressed pregnant women: an exploratory analysis of pregnancy outcomes. Psychosom Med 2013;75:670-81.

14. Okun ML, Schetter CD, Glynn LM. Poor sleep quality is associated with preterm birth. Sleep 2011;34:1493-8.

15. Lee KA, Zaffke ME, McEnany G. Parity and sleep patterns during and after pregnancy. Obstet Gynecol 2000;95:14-18.

16. Drager LF, Togeiro SM, Polotsky VY, et al. Obstructive sleep apnea a cardiometabolic risk in obesity and the metabolic syndrome. J Am Coll Cardiol 2013;62:569-76.

17. Guidolin M, Gradisar M. Is shortened sleep duration a risk factor for overweight and obesity during adolescence? A review of the empirical literature. Sleep Med 2012;13:779-86.

18. Knutson KL. Does inadequate sleep play a role in vulnerability to obesity? Am J Hum Biol 2012;24:361-71.

19. Zimberg IZ, Damaso A, Del Re M, et al. Short sleep duration and obesity: mechanisms and future perspectives. Cell Biochem Funct 2012;30:524-9.

20. Cuellar NG. The effects of periodic limb movements in sleep (PLMS) on cardiovascular disease. Heart Lung 2013;42:353-60.

21. Dong JY, Zhang YH, Qin LQ. Obstructive sleep apnea and cardiovascular risk: meta-analysis of prospective cohort studies. Atherosclerosis 2013;229:489-95.

22. Kasai T, Floras JS, Bradley TD. Sleep apnea and cardiovascular disease: a bidirectional relationship. Circulation 2012;126:1495-510.

23. Stopford E, Ravi K, Nayar V. The association of sleep disordered breathing with heart failure and other cardiovascular conditions. Cardiol Res Pract 2013;2013:356280.

24. Lopresti AL, Hood SD, Drummond PD. A review of lifestyle factors that contribute to important pathways associated with major depression: diet, sleep and exercise. J Affect Disord 2013;148:12-27.

25. Palagini L, Baglioni C, Ciapparelli A, et al. REM sleep dysregulation in depression: state of the art. Sleep Med Rev 2013;17:377-90.

26. O'Brien LM, Bullough AS, Owusu JT, et al. Pregnancy-onset habitual snoring, gestational hypertension, and preeclampsia: prospective cohort study. Am J Obstet Gynecol 2012;207:487.e1-9.

27. Stacey T, Thompson JM, Mitchell EA, et al. Association between maternal sleep practices and risk of late stillbirth: a case-control study. BMJ 2011;342:d3403.

28. Strange LB, Parker KP, Moore ML, et al. Disturbed sleep and preterm birth: a potential relationship. Clin Exp Obstet Gynecol 2009;36:166-8.

29. Micheli K, Komninos I, Bagkeris E, et al. Sleep patterns in late pregnancy and risk of preterm birth and fetal growth restriction. Epidemiology 2011;22:738-44

30. Loube DI, Poceta JS, Morales MC, et al. Self-reported snoring in pregnancy. Association with fetal outcome. Chest 1996;109:885-9.
31. O'Brien LM, Bullough AS, Owusu JT, et al. Snoring during pregnancy and delivery outcomes: a cohort study. Sleep 2013;36:1625-32.

32. Mantell CD, Craig ED, Stewart AW, et al. Ethnicity and birth outcome: New Zealand trends 1980-2001: Part 2. Pregnancy outcomes for Maori women. Aust N Z J Obstet Gynaecol 2004;44:537-40.

33. Paine SJ, Priston M, Signal TL, et al. Developing new approaches for the recruitment and retention of Indigenous participants in longitudinal research: Lessons from E Moe, Māmā: Maternal Sleep and Health in Aotearoa/New Zealand. MAI J 2013;2: 121-32.

34. Gallo AM, Lee KA. Sleep characteristics in hospitalized antepartum patients. J Obstet Gynecol Neonatal Nurs 2008;37:715-21.

35. Lee KA, Gay CL. Can modifications to the bedroom environment improve the sleep of new parents? Two randomized controlled trials. Res Nurs Health 2011;34:7-19.

36. 2007 Women and Sleep. http://sleepfoundation.org/sleep-polls-data/ sleep-in-america-poll/2007-women-and-sleep

37. Johns MW. A new method for measuring daytime sleepiness: the Epworth sleepiness scale. Sleep 1991;14:540-5.

38. Buysse DJ, Reynolds CF III, Monk TH, et al. The Pittsburgh Sleep Quality Index: a new instrument for psychiatric practice and research. Psychiatry Res 1989;28:193-213.

39. Ekbom K, Ulfberg J. Restless legs syndrome. J Intern Med 2009;266:419-31.

40. McCowan L, Stewart AW, Francis A, et al. A customised birthweight centile calculator developed for a New Zealand population. Aust $N Z$ J Obstet Gynaecol 2004:44:428-31.

41. McCowan L, Groom K, Stewart A. The use of customised versus population-based birthweight standards in predicting perinatal mortality. BJOG 2007;114:1302-3; author reply 1303.

42. McCowan LM, Harding JE, Stewart AW. Customized birthweight centiles predict SGA pregnancies with perinatal morbidity. BJOG 2005;112:1026-33.

43. World Health Organization. The ICD-10 classification of mental and behavioural disorders: clinical descriptions and diagnostic guidelines. Geneva: World Health Organization, 1992.

44. Paine S-J, Gander PH. Sleep, sleepiness and sleep disorders: principles for examining differences by ethnicity. In: Kushida C, ed. The encyclopedia od sleep. Vol 2. Waltham, MA: Academic Press, 2013:691-8

45. Salmond C, Crampton P, Atkinson J. NZDep2006 Index of Deprivation User's Manual. Wellington, New Zealand: Department of Public Health, University of Otago, Wellington, 2007.

46. Cox J, Holden J, Sagovsky R. Detection of postnatal depression: the development of the 10-item Edinburgh Postnatal Depression Scale. Br J Psychiatry 1987;150:782-6.

47. Fung AM, Wilson DL, Lappas M, et al. Effects of maternal obstructive sleep apnoea on fetal growth: a prospective cohort study. PLOS ONE 2013;8:e68057.

48. Kennelly MM, Fallon A, Farah $\mathrm{N}$, et al. Effects of body mass index on sleep patterns during pregnancy. J Obstet Gynaecol 2011;31:125-7.

49. Paine SJ, Gander PH, Harris R, et al. Who reports insomnia? Relationships with age, sex, ethnicity, and socioeconomic deprivation. Sleep 2004;27:1163-9.

50. Paine SJ, Gander PH, Harris RB, et al. Prevalence and consequences of insomnia in New Zealand: disparities between Maori and non-Maori. Aust N Z J Public Health 2005;29:22-8.

51. Reducing inequalities in health. http://www.health.govt.nz/ publication/reducing-inequalities-health

52. New Zealand Health Survey: annual update of key findings 2012/13. http://www.health.govt.nz/publication/new-zealand-health-surveyannual-update-key-findings-2012-13 